\title{
Analysis of Signal Impairment and Crosstalk Penalty Induced by Different Types of Optical Filters in 100 Gbps PM-DQPSK Based Systems
}

\author{
Xiaoyong Chen, Paloma R. Horche, Alfredo Martín Minguez
}

\begin{abstract}
Optical filters are crucial elements in optical communication networks. Their influence toward the optical signal will affect the communication quality seriously. In this paper we will study and simulate the optical signal impairment and crosstalk penalty caused by different kinds of filters, which include Butterworth, Bessel, Fiber Bragg Grating (FBG) and Fabry-Perot (F-P). Signal impairment from filter concatenation effect and crosstalk penalty from out-band and in-band are analyzed from Q-penalty, eye opening penalty (EOP) and optical spectrum. The simulation results show that signal impairment and crosstalk penalty induced by the Butterworth filter is the minimum among these four types of filters. Signal impairment caused by filter concatenation effect shows that when center frequency of all filters is aligned perfectly with the laser's frequency, 1250 GHz Butterworth filters can be cascaded, with 1-dB EOP. This value is reduced to 9 when the center frequency is misaligned with $5 \mathrm{GHz}$. In the $50-\mathrm{GHz}$ channel spacing DWDM networks, total Q-penalty induced by a pair of Butterworth filters based demultiplexer and multiplexer is lower than $0.5 \mathrm{~dB}$ when the filter bandwidth is in the range of 42-46 GHz.
\end{abstract}

Keywords-optical filter; optical communication; polarization multiplexed differential quadrature phase shift keying (PM-DQPSK); signal impairment; optical crosstalk

\section{INTRODUCTION}

Optical networks are designed to keep optical signals in the optical domain for as long as possible before their signal quality has been aggravated to the level at which optical-to-electrical-to-optical (O/E/O) regeneration is necessary. In such a way, optical cross-connects (OXCs) and reconfigurable optical add-drop multiplexers (ROADMs) are used to adding, dropping and switching channels from one optical fiber to another one in the network nodes. Usually, the OXCs and ROADMs comprise of several filters, such as wavelength blockers (WB) and wavelength selective switches (WSS). In addition, WDM multiplexers and demultiplexers used in the terminals and Dispersion Compensation Modules (DCMs), which are based on chirped fiber Bragg gratings, used in channels also contain one or more filters. Thus, the filters' performance will affect the signal quality seriously, especially in the long-haul transmission networks. Due to the undesirable design, the filters will not only induce optical crosstalk from the neighboring channels, but also lead to filter concatenation effect when the signals pass through several filters. Furthermore, when the center frequency of the filters does not align to the optical signal frequency, signal distortion and crosstalk induced by filters will be further raised, leading to signal quality further deteriorated.

Many previous papers have studied the effects caused by filters in optical communication networks. In [5], the performance of a cascade of 11 filters with bandwidth of $50 \mathrm{GHz}$ has been studied in a $20 \mathrm{~Gb} / \mathrm{s}$ NRZ system. In [3, $4,6]$ signal impairment caused by filter concatenation in coherent optical OFDM system has been presented. Simulation of signal impairment caused by cascaded ROADMs has been shown in [7]. Signal distortion and crosstalk penalties induced by optical filters in 10 Gbps NRZ/RZ based optical networks have been commented in [8]. Analysis of loss ripple, group delay ripple and dispersion induced by optical filters have presented in [9, $10,11]$. However, almost all the papers mentioned above just discuss the performance of one kind of filters. In optical communication system, there are many types of optical filters, such as Butterworth optical fiber [8, 12], Gaussian optical filter [7], Bessel optical filter [13], fiber Bragg grating (FBG) [14], Fabry-Perot optical filter [14] and so on. In [12], we have presented signal impairment caused by different types of filters in 40 Gbps DQPSK and 100 Gbps PM-DQPSK systems with only one channel. In this work, we will study the signal impairment and crosstslk induced by different types of filters in $100 \mathrm{Gbps}$ PM-DQPSK based DWDM networks. Given the $50 \mathrm{GHz}$ ITU grid based infrastructure used today, in order to improve the capacity, one could consider replacing step by step the 10 Gbps NRZ channels by the 40 Gbps DQPSK or $100 \mathrm{Gbps}$ PM-DQPSK ones as a function of the capacity needed [15]. That means before all channels are replaced 
by the 100 Gbps PM-DQPSK channels, there are two or more types of channels existing in the same transmission system. However, in this paper we only study the signal impairment and crosstalk penalty induced by different types of filters in the 100 Gbps PM-DQPSK based networks.

The paper is organized as follows. Signal impairment caused by different types of filters is presented in Section II, while in-band and out-band crosstalk penalties induced by different types of filters are discussed in Section III. In Section IV, simulation results of total Q-penalty induced by an OXC are presented. Finally, we present the conclusion in Section V.

\section{FILTER CONCATENATION EFFECT}

\section{A. Characteristics of different filters}

As mentioned previously, in a Wavelength Division Multiplexing (WDM) optical network, an optical signal will pass through many optical nodes before reaching the destination. Those nodes, which in fact comprise of several filters, will cause filter concatenation effect, leading to signal quality aggravated. That is because the filters don't have ideal transfer functions for both optical amplitude and phase. With optical filters increasing in the channel, filter concatenation effect will be more serious.

The effective transfer function of cascaded filters is the product of every individual filter. Therefore, the effective transmission bandwidth of cascaded filters is much narrower than that of an individual filter. Moreover, when the center frequency of the cascaded filters is misalignment, it will further narrow the effective bandwidth. This effect can be seen from Fig. 1. Compared with an individual $3^{\text {rd }}$ order Butterworth filter, the effective bandwidth of 10 cascaded Butterworth filters is much narrow (red line), even if these 10 filters are aligned in the center frequency. When these 10 filters are cascaded with center frequency misalignment of $\pm 5 \mathrm{GHz}$, the effective bandwidth is further

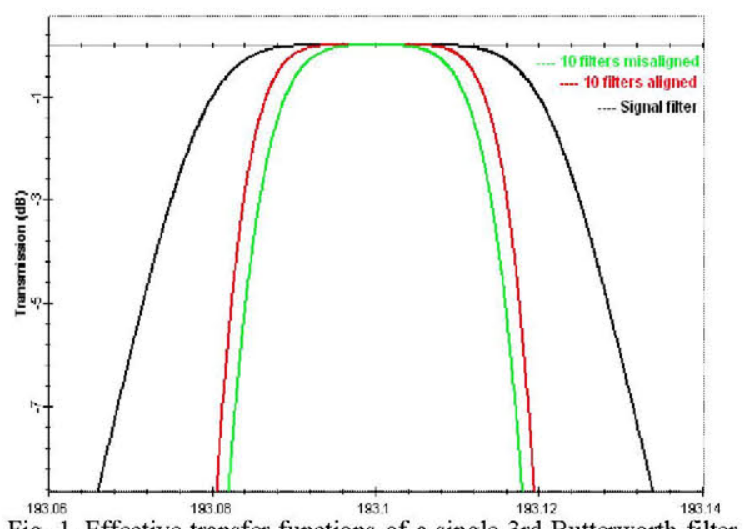

Fig. 1. Effective transfer functions of a single $3 \mathrm{rd}$ Butterworth filter and 10 cascaded filters aligned (red) and misaligned (green)

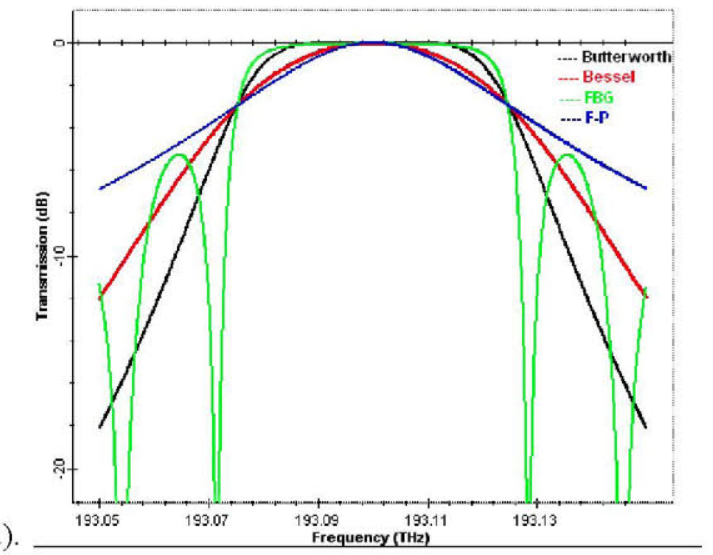

a).

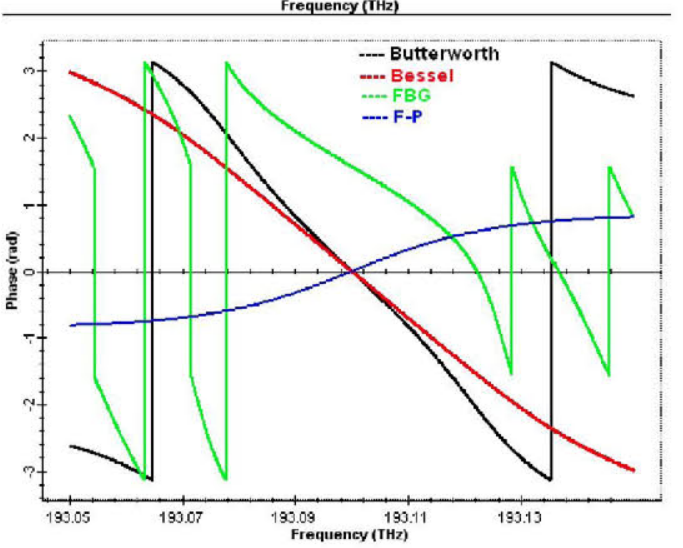

b).

Fig. 2. (a) Amplitude transfer function of four kinds of filters; (b) phase transfer function of four kinds of filters

narrowed. This can be seen in the green line of Fig. 1. That is because the transfer function of the filter is not an ideal rectangle transfer function. With the number of cascaded filters increasing, the flat top region the effective transfer function becomes smaller and the tail decay becomes steeper. This makes the effective bandwidth narrower, especially when the cascaded filters are not aligned perfectly.

However, different types of filters will lead to different effects toward the signals. This can be explained from the amplitude and phase transfer functions, as shown in Fig. 2. We can see that the F-P filter has the flattest transmission spectrum and it also has large phase linear region. Combining these two factors it can be known that the F-P filter has less effect to the signal in the individual condition. However, since F-P filter has smallest flat top region in the transmission spectrum, it decides that the effective bandwidth will decrease seriously with the cascaded number increase, as shown in Fig. 3. The size of flat top region of Bessel filter is a little larger than F-P filter and it also has phase linear region as large as F-P filter. Thus, it can be derived that the effective bandwidth of cascaded Bessel filters is larger than that of the F-P 


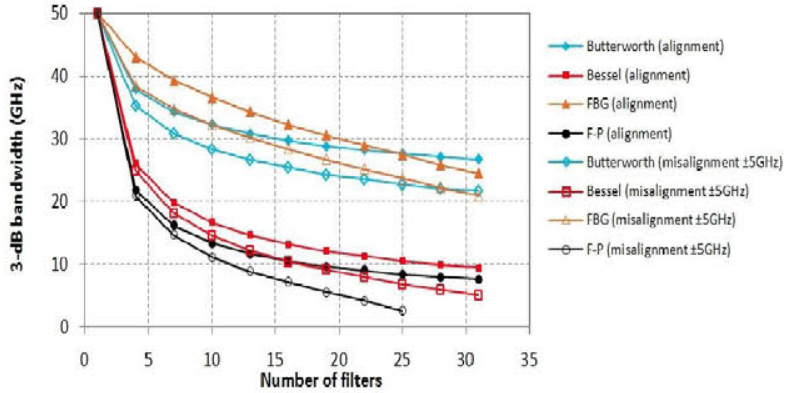

Fig. 3. 3-dB bandwidth as a function of number of filters for different kinds of filters

filters in the same condition, as shown in Fig. 3. In addition, we can also derive that the performance of cascaded Bessel filters is better than that of F-P filters. This can be demonstrated in the simulation, as the simulation results shown in Fig. 5. Fig. 2a also shows that the Butterworth filter and FBG have wide flat top region. This makes the effective bandwidth decreases slowly when the number of cascaded filters increases. However, compared with Bessel and F-P filter, the phase linear region of the Butterworth filter and FBG is narrower. The effective linear region will further narrow when filters are cascaded, leading to signal quality further being aggravated.

\section{B. Simulation in a single 100 Gbps PM-DQPSK channel}

In order to make the simulation structure much simple and close to the real conditions, we design a simulation scheme as shown in Fig. 4. The modulation formats used in the simulation is $100 \mathrm{Gbps}$ PM-NRZ-DQPSK. The loop controller is used to decide how many times the signal passes through the filter. In the scheme the erbium-doped fiber amplifiers (EDFAs) are used to compensate the insertion loss and attenuation introduced by single mode fiber (SMF) and dispersion compensating fiber (DCF). The DCF is used to compensate the accumulated dispersion generated by the SMF. In order to avoid introducing nonlinear effects, the power laughed into the fiber is kept lower than $-4 \mathrm{dBm}$. Then the signal goes into a 10: 90 power splitter to divide into two beams. One goes to the

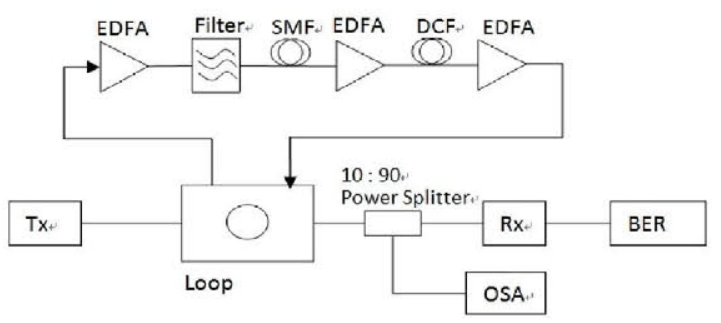

Fig. 4. Simulation setup: Tx: PM-(NRZ)-DPQSK Transmitter; Rx: direct detection PM-DPQSK Receiver; OSA: Optical Spectrum Analyzer; BEI Analyzer: Bit Error Rate Analyzer; EDFA: Erbium-doped fiber amplifier SMF: single mode fiber; DCF: dispersion compensation fiber.

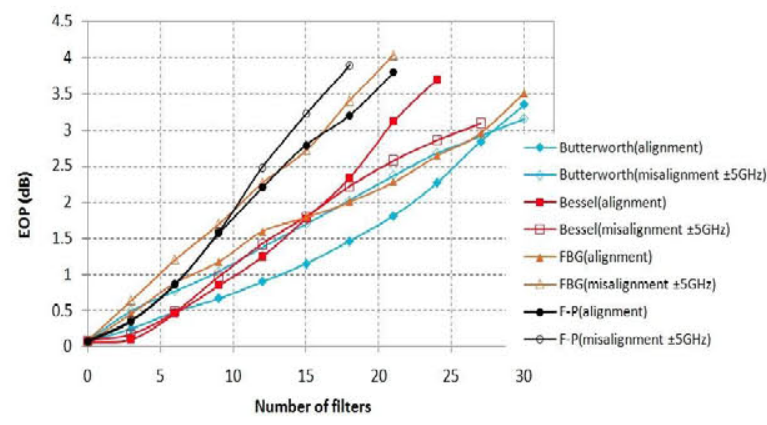

Fig. 5. EOP as a function of cascaded filters in the $100 \mathrm{Gbps}$ PMNRZ-DQPSK system with (a) all the filters' center frequency aligned and (b) filters' center frequency misaligned of $\pm 5 \mathrm{GHz}$

optical spectrum analyzer for observing the signal spectrum. The other one goes to the receiver. Finally a bit error rate (BER) analyzer is connected to the receiver to analyze the performance of the received signal. Since the signal power propagating in the fibers is lower than $4 \mathrm{dBm}$, we don't consider the nonlinear effects in the simulation.

In this section we analyze the signal impairment from eye-opening penalty (EOP). The eye opening parameter is defined as the intensity difference between the minimum "ones" value and the maximum "zeros" value without noise. The EOP is defined as the difference between the normalized eye opening after passing through an optical link without any filters and the normalized eye opening after passing through the same optical link with a given number of filters [8]. The eye opening parameter and EOP can be described by the following functions, respectively [16].

$$
\begin{aligned}
& \text { Eye Opening }=V_{1}-V_{0} \\
& E O P=10 \log \left(\frac{V_{u, 1}-V_{u, 0}}{V_{d, 1}-V_{d, 0}}\right)
\end{aligned}
$$

where $V_{1}$ is the voltage level of the minimum " 1 " rail at the eye center, and $V_{0}$ is the voltage level of the maximum " 0 " rail. The subscript " $u$ " represents the undistorted state of the signal and " $d$ " represents the distorted state of the signal.

The simulation results are shown in Fig. 5. When all filters are aligned perfectly with the laser's central frequency, the Butterworth filter shows the best performance among these four types of filters. With $1 \mathrm{~dB}$ EOP, about 12 Butterworth filters can be cascaded in the channel, while the permitted cascaded number for FBG, F$\mathrm{P}$ and Bessel filter are 6,6 and 10, respectively. When all filters are randomly misaligned with the laser's central frequency $( \pm 5 \mathrm{GHz})$, signal impairments caused by FBG 


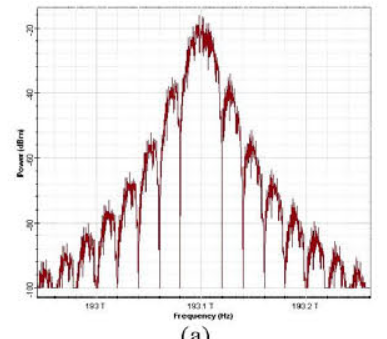

(a)

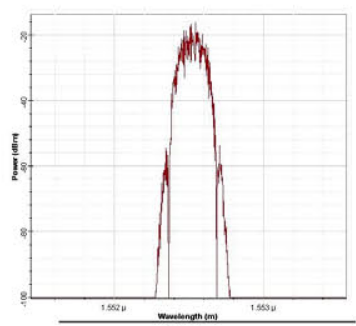

(b)
Fig. 6. Signal Spectrum (a) after $13^{\text {rd }}$ order Butterworth filter; (b) after 18 $3^{\text {rd }}$ order Butterworth filters

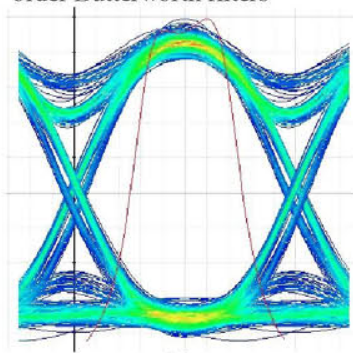

(a)

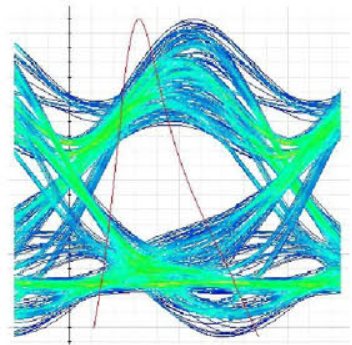

(b)
Fig. 7. Eye diagrams, (a) after $13^{\text {rd }}$ order Butterworth filter; (b) after 18 $3^{\text {rd }}$ order Butterworth filters concatenation.

and Butterworth filters concatenation become much more serious. Only 5 FBG and 9 Butterworth filters can be cascaded with $1 \mathrm{~dB}$ EOP. The Bessel filter shows a better performance. In the limitation of $1 \mathrm{~dB}$ EOP, 9 Bessel filters can be cascaded. The results also show that $6 \mathrm{~F}-\mathrm{P}$ can be cascaded either in the situation of all filters aligned or not. The effect of signal spectrum clipping caused by filters concatenation can be seen in Fig. 6 and the signal distortion in time domain can be seen from eye diagrams shown in Fig. 7.

\section{CROSSTALK}

Given that the filters are not ideal, some power will be sent to the wrong channels in the optical nodes, as shown in Fig. 8. This leaked power will not only generate outband crosstalk, but also induce in-band crosstalk. These two types of crosstalks will take penalties to the signals, especially the latter. In this section we will analyze the signal penalties casused by these two crosstalks from a Qpenalty, which can be defined as the following function:

$$
Q-\text { penalty }=20 \log \left(E_{1} / E_{2}\right)
$$

where $E_{1}$ and $E_{2}$ are eye opening percentages without and with filters, respectively.

\section{A. Out-band crosstalk}

Out-band crosstalk is the linear crosstalk suffered at the receiver by leakage of some power from adjacent channels into the main signal to be detected. This type of crosstalk only occurs at the final drop location of the channel, the crosstalk terms are different wavelength from the main signal. Usually, the leaked power from adjacent channels is

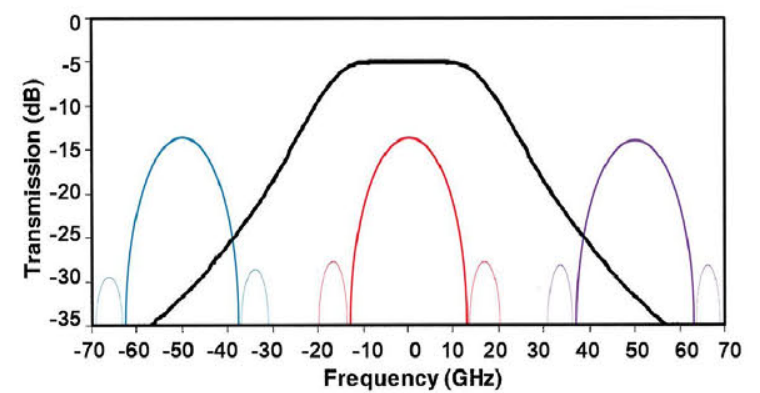

Fig. 8. Transmission function of an optical filter and the origin of crosstalk

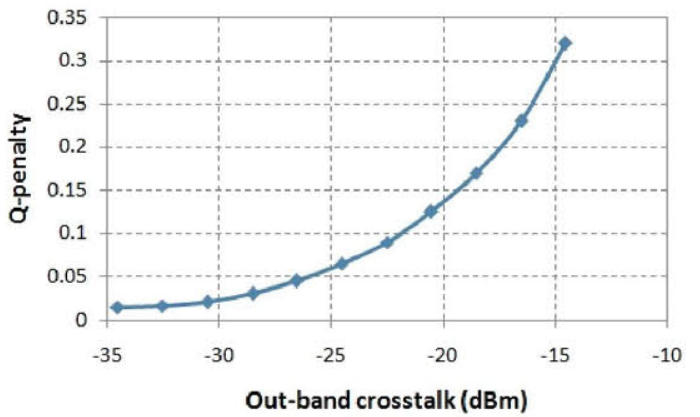

Fig. 9. Q-penalty as a function of out-band crosstalk

very small. Therefore, signal penalty induced by this type of crosstalk is not serious. In order to see the relationship between the Q-penalty and the out-band crosstalk, we can change the value of out-band crosstalk by changing the adjacent channel power. The Q-penalty caused by the outband crosstalk is shown in Fig. 9. It can be see that the Qpenalty is very small when the out-band crosstalk is lower than $-20 \mathrm{dBm}$.

\section{B. In-band crosstalk}

In-band crosstalk is well recognized as a potentially serious impairment in optical networks with optical switching and configuration nodes, such as OXCs and ROADMs [8]. This type of crosstalk occurs between the signal and the leaked signals which have the same wavelength. In-band crosstalk can also be generated within an optical node by the leakage of small amounts of signal power into different output ports of a demultiplexer (DEMUX) and then further leakage of those small signal copies into the outgoing transmission fiber by the multiplexer (MUX) at the node output side. The in-band crosstalk caused by leaked power is decided by the effective bandwidth and the effective transmission function of a pair of filters used in the DEMUX and MUX. Thus, different types of filters can induce different leakage, leading to different in-band crosstalk. Fig. 10 shows crosstalk power leaked through an adjacent channel port for a pair of DEMUX and MUX with a $100 \mathrm{Gbps}$ PMDQPSK signal. It is normalized to the transmission value at the filter center frequency. It shows that the Butterworth filter generate the least leakage among these four types of filters, while the leakage induced by the F-P is the maximum. 
According to (3), Q-penalty can be calculated from the eye-opening percentages in different conditions. We first calculate eye-opening percentage for a given system in the absence of crosstalk. Then a new eye-opening percentage which is affect by the crosstalk is calculated. The difference between these two values is the Q-penalty due to the crosstalk. The calculation results are shown in Fig. 11. It can be seen that the in-band crosstalk is corresponding to the leaked power. With the bandwidth increase, leaked power increases, leading to Q-penalty sharply increase. The results also show that the Butterworth filter based DEMUX and MUX has the best performance. When the bandwidth is narrower than 44 $\mathrm{GHz}$, the Q-penalty induced by in-band crosstalk is lower than $0.1 \mathrm{~dB}$.

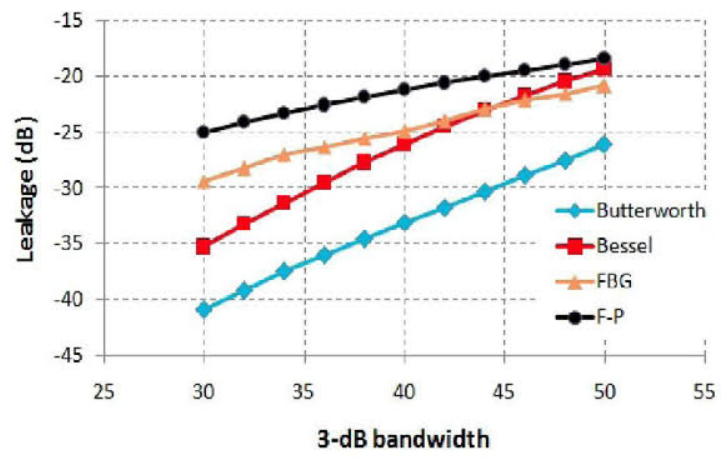

Fig. 10. Crosstalk power level as a function of 3-dB bandwidth

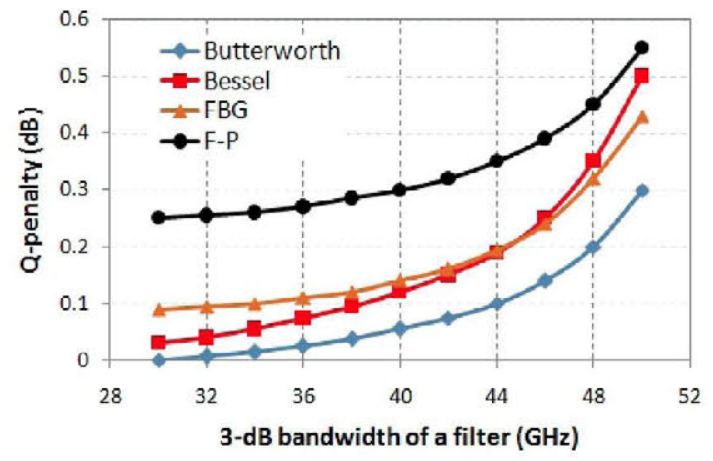

Fig. 11. Q-penalty induced by In-band crosstalk as a function of the bandwidth of the filter used in the DEMUX/MUX

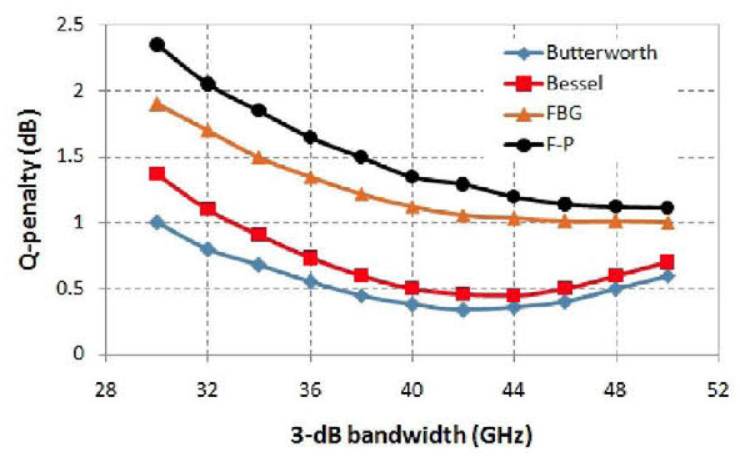

Fig. 12. Total Q-penalty induced by a OXC based on different types of filters

\section{SIMULATION RESULTS}

Given the signal impairment and crosstalk penalties analyzed above, we build an optical system to observe the total Q-penalty caused by an OXC which is based on different types of filters. The system contains $550-\mathrm{GHz}$ channels and the signals used in the simulation are the 100 Gbps PM-DQPSK signals. The simulation results are shown in Fig. 12. From the simulation results we can see that the Q-penalty induced by F-P and FBG is much larger than that caused by Bessel and Butterworth filters. The results also show that when the bandwidth of Butterworth and Bessel filters are in the range of $40-46 \mathrm{GHz}$, the total Q-penalty is the smallest, with only about $0.5 \mathrm{~dB}$ Qpenalty. Here we have to point out that the simulation results shown in this section are based on all filters aligned. When all filters are not perfectly aligned, much more Qpenalty will be generated.

\section{CONCLUSION}

In this paper we study and simulate signal impairment and crosstalk penalty induced by different kinds of filters. The theoretical analysis and simulation results show that the Butterworth filter has the best performance among these four types of filters. In the single 100 Gbps PMDQPSK channel, 12 Butterworth filters can be cascaded in the condition of center frequency aligned and 9 for center frequency misaligned with $\pm 5 \mathrm{GHz}$. Study of crosstalk shows that in-band crosstalk will seriously affect the signal quality. Crosstalk induced by Butterworth filter is the smallest among these four types of filters. When the bandwidth is narrower than $44 \mathrm{GHz}$, in-band crosstalk induced by a pair of Butterworth filters based DEMUX and MUX is lower than $0.1 \mathrm{~dB}$. Finally, the optical network simulation shows that the total Q-penalty induced by an OXC, which is based on a pair of Butterworth or Bessel filters, can be lower than $0.5 \mathrm{~dB}$ when the filter bandwidth is in the range of $40-46 \mathrm{GHz}$.

\section{ACKNOWLEDGMENT}

The authors would like to acknowledge support from the China Scholarship Council (CSC).

\section{REFERENCES}

[1] S. Sygletos, A. Tzanakaki and I. Tomkos, "Numerical Study of Cascadability Performance of continuous Spectrum Wavelength Blocker/Selective Switch at 10/40/160 Gb/s," IEEE Photonics Technology Letters, vol. 18, pp. 2608-2610, 2006

[2] J. D. Downie, I. Tomkos, N. Antoniades and A. Boskovic, "Effects of Filter Concatenation for Directly Modulated Transmission Lasers at 2.5 and $10 \mathrm{~Gb} / \mathrm{s}$," J. Lightwave Technology, vol. 20, pp. 218-227, 2002

[3] Y. Tang and W. Shich, "Filter concatenation impact on $107-\mathrm{Gb} / \mathrm{s}$ coherent optical OFDM system," in Proc. OECC, p. FQ2, 1-2, 2009

[4] E. Giacounidis, I. Tomkos, and J. M. Tang, "Adaptive ModulationInduced Reduction in Filter Concatenation Impairment for Optical OFDM Metro/Regional Systems," J. Opt. Commun. Netw., vol. 3, pp. 587-593, 2011 
[5] D. M. Pataca, J. C. R. F. Oliveira, A. A. Juriollo and A. F. Herbster, "Transmission of a $20 \mathrm{~Gb} / \mathrm{s}$ NRZ OOK Signal Throughout a $390 \mathrm{~km}$ Fiber Link and a Cascade of $10 \times 50 \mathrm{GHz}$ Filters and $9 \times$ EDFAs," J. Microwaves Optoelectronics and Electromagnetic Applications, vol. 10, pp. 143-154, 2011

[6] M. Chochol, J. M. Fabrega, M. S. Moreolo and G. Junyent, "Optical Filter Cascading Effects in a Phase Modulated Coherent Optical OFDM Transmission System Based on Hartley Transform," in Proc. ICTON, p. Tu.P.3, 2012

[7] S. Tibuleac and M. Filer, "Transmission Impairments in DWDM Networks with Reconfigurable Optical Add-Drop Multiplexers," J. lightwave Technology, vol. 28 , pp. 557-568, 2010

[8] J. D. Downie and A. B. Ruffin, "Analysis of Signal Distortion and Crosstalk Penalties Induced by Optical Filters in Optical Networks," J. Lightwave Technology, vol. 21, pp. 1176-1186, 2003

[9] X. Liu, X. Wei, A. H. Gnauck, C. R. Doerr and S. Chandrasekhar, "Analysis of Loss Ripple and Its Application to the Mitigation of Optical Filtering Penalty," IEEE Photonics Technology Letters, vol. 17, pp. 82-84, 2005

[10] H. Chotar, Y. Painchaud, A. Mailloux, M. Morin, F. Trepanier and M. Guy, "Group delay Ripple of Cascaded Bragg Grating Gain
Flattening Filters," IEEE Photonics Technology Letters, vol. 14, pp $1130-1132,2002$

[11] G. Lenz, B. J. Eggleton, C. K. Madsen, C. R. Giles and G. Nykolak, "Optimal Dispersion of Optical Filters for WDM Systems," IEEE Photonics Technology Letters, vol. 10, pp. 567-569, 1998

[12] X. Chen, P.R. Horche, A.M. Minguez, "Optical signal impairment study of cascaded optical filters in $40 \mathrm{Gbps}$ DQPSK and $100 \mathrm{Gbps}$ PM-DQPSK systems," in Proc., SPIE 2013, San Diego, CA, p. $8855 \mathrm{C}, 2013$

[13] S. Takeda and Y. Shigeoka, "An optical thin film Bessel filter for 40 Gbit/sec-100GHz spacing D-WDM system," in Proc, ECOC 2002 Gopenhagen, Denmark, p. PD 2.9, 2002

[14] G. P. Agrawal, "Fiber-Optic Communication Systems," $4^{\text {th }}$ ed., Wiley, New Jersey, 2010, pp. 232-243

[15] E. Pincemin, J. Karaki, Y. Loussouarn, H. Poignant, C. Betoule, G. Thouenon and R. L. Bidan, "Challenges of 40/100 Gbps and higherrate deployments over long-haul transport networks," Optical Fiber Technology, vol. 17, pp. 335-362, 2011

[16] J. D. Downie, "Relationship of Q Penalty to Eye-Closure Penalty for NRZ and RZ Signals With Signal-Dependent Noise," J. Lightwave Technology, vol. 23, pp. 2031-2038, 2005 